V.

Von der chirurgisehen Abtheilung des Bürgerhospitals zu Köln.

\title{
Ein Beitrag zur conservativen Behandlung complicirter Verletzungen der Extremitäten.
}

(Im Auszug vorgetragen auf der diesjährigen Aerzteversanımlung )

Von

Dr. Plücker,

Secundararzt.

(Mit à Abbildungen.)

Bei Nachprufung der Untersuchungen Sehimmelbus ch's über die Wundinfeetion kamen andere Autoren zu dem Ergebniss, dass die Infection, wenn nicht abnorm ungünstige Verhältnisse vorlagen, zunäehst eine örtliche war; dass eine gewisse Zeit zur Anpassung des aus Sehmutz und Staub in die Wunden gelangten Keimmaterials vergehen musste, ele eine fortschreitende Infeetion erfolgte. Ls gelang Nalba n, Noctze l, Friedrich, durch frülzeitiges Eliminiren der infieirten Stelle eine Allgemeininfection zu verhindern. Parallele Studien am verletzten Mensolen ergaben Frie d rich mindestens gleieh günstige Resultate. Dies veranlasste ihn zu der Sehlussfolgerung, dass die rechtzeitige Anfriselung als das Ideal zur Verhütung von Wundinfection anzusehen ist; er bezeichnet dieselbe als radicale aber einzige Maassnahme, welehe die Infectionsgefahr beseitigen kann. - Wir wïrden bei solehem Vorgehen zumeist in der Lage sein, uns aseptisehe Wundverhältnisse zu schaffen; ausgenommon wären diejenigen Fälle, die nielit im Verlauf der ersten Stunden in die Behandlung treten, sowie diejenigen selteneren Fälle, bei welchen grössere Mengen pathogenen Materials oder hoch virulente Keime Eingang gefunden haben.

Da a priori jede Wundverletzung als möglicherweise inficirt anzusehen ist, so würde die rigorose Befolgung des Postulats, reine Wundverhältnisse zu schaffen, in jedem Falle olne Rücksicht durchzuführen sein. Wir würden dazu kommen, z. B. bei grösseren Quetseh- 
Risswunden des Gesichts und der Finger Defecte zu setzen, deren Heilresultat uns nicht befriedigen dürfte. Wie sollte es mit den Stich- und Schussverletzungen werden, wie mit den complicirten Fracturen einfacher Art? Dazu kommt, dass ein streng aseptisebes Vorgehen bei jeder einzelnen Wundverletzung, sowohl im poliklinisehen Betriebe als auch in der privatärztlichen Praxis auf erhebliche Schwierigkeiten stossen würde. Glücklicherweise hat die bisher wohl meist gelibte conservative Behandlung kleinerer Wundrerletzungen so befriedigende Resultate zu verzeichnen, dass wir keine Veranlassung haben, die einfache Methode der Complicirten zu opfern. - Abgesehen von klaffenden Gesichtswunden und Sehnendurchtrennungen, deren primäre Vereinigung immer zu versuchen ist, wurden im Kölner Bürgerhospital bis zum Jabre 1896 die kleineren Wundverletzungen nach Reinigung der Umgebung einfach aseptisch verbunden oder mit einem antiseptiseben Pulver bestäubt, bei schon entzündeter Umgebung wurden feuchte antiseptische Verbände angewandt. - Seit 1896 hatten wir, veranlasst durch die bekannten Veröffentlichungen Lockwood's, Lauenstein's u. a., über die Möglichkeit einer 2. Nabtinfection von der Haut aus begonnen, die aseptische Naht mit einem Antisepticum zu bedecken. Wir bedienten uns anfänglich der Silbergaze, um dann seit Fruhjahr 1897 zur Anwendung einer 25 proc. Hydrg. ox.-flav. Salbe uberzugehen, welche M ajew ski auf Grund grösserer Versuchsreihen eindringlich empfohlen hatte . Eine ausgedehnte Verwendung fand diese Salbe in der Poliklinik; annähernd 1500 Wundverletzungen aller Art wurden in der einfachen Weise behandelt, dass auf aseptiseher Gaze eine dünne Schicht gelber Salbe aufgetragen und damit die Wunde bedeckt wurde; nur bei grober Verunreinigung wurde die Umgebung gesäubert. - Niemals trat cine Complication auf. Von der Anwendung des Mittels auf grosse Wundfächen haben wir keine Nachtheile gesehen; eczeme Quecksilbererschein ungen wurden vereinzelt beobachtet. - Den günstigen Heilverlauf schreiben wir bauptsächlich der Vermeidung jeglieher Irritation der Wunden zu; wahrscheinlich ist ja, dass, soweit das Antisepticum mit inficirten Partien in Berührung tritt, die Entwickelung gewöhnlicher pathogener Keime aufgehalten wird.

Wie die einfachen Wundverletzungen die besten Resultate geben, wenn wir möglichst wenig daran machen, so gilt dies in höherem Grade für die Behandlung der einfachen complicirten Fracturen. Seit Jahren wurde sie bei uns derart gellbt, dass nach Reinigung der Wundumgebung ein aseptischer Verband angelegt und die corrigirten Fragmente im Gypsverband fixirt wurden. Der Gypsverband 
umfasste immer die ganze Extremität und bei complicirten Oberarm-, bezw. Oberschenkelfracturen immer den Schulter-, bezw. Beckengürtel; nur so schien eine völlige Ruhestellung gesichert. Thunlichst wurden alle derartige Fälle chloroformirt, und zwar vor Abnahme der provisorisehen Verbände, um unwillktirliche Bewegungen und unvermeidliche Zerrungen möglichst auszuschalten, und um andererseits die Anlegung eines correcten Verbandes zu erleichtern. Wir modificirten unser Vorgehen dahin, dass wir nur noch bei grober Verunreinigung der Umgebung eine Reinigung vornehmen unter Schutz der Wunde, uns sonst aber darauf beschränken, die verletzte Stelle mit einem Verband von Hydrg. ox.-flav. Salbe zu bedecken. Es lagen auf diese Weise Verhältnisse vor wie bei einer subcutanen Fractur; ein Postulat, welehes meines Wissens Herr Geheimrath v. Bergmann vor Jahren schon für die Behandlung einfacher complicirter Frakturen aufgestellt hat. In keinem Falle von einfacher complicirter Fraktur hatten wir Misscrfolg zu verzeichnen.

Gegrenuiber diesen einfachen Verhältnissen gestaltet sich die Beurtheilung und Behandlung schwerer complicirter Verletzungen ausserordentlich sehwierig; es sind diejenigen Fälle, in denen neben einfachem oder gesplittertem Knochenbruch grosse Weichtheilquetschungen, Zerreissungen und Quetsehungen stattgefunden haben; besonders gefährlich bei gleichzeitiger Eröffnung benachbarter Gelenke. - Es erscheint zweckmässig, die schweren complicirten Verletzungen in der Continuität der langen Röhrenknochen von den peripheren zu trennen. - Die gewöhnliche Ursache der ersteren sind Ueberfahrung, Maschinengewalt, Sturz aus grosser Höhe, Schusswirkung. Derartige Verletzungen werden in der Regel den Kliniken und grossen Krankenhäusern direct von der Unfallsstelle zugefthhrt; nicht ganz selten gelangen sie auch nach längerem Transport in die Behandlung. Wir wissen nicht, in wie weit unmittelbar nach der Verletzung Störungen der Wunde verursacht wurden; die Entfernung aus der gefähırdeten Lage nach dem Unglüek, die Anlegung des provisorischen Verbandes, der sich auch äratlicherseits zumeist auf einfaches Zudecken mit antiseptischer Gaze und Schienen beschränken wird, lassen Zerrungen und Quetschungen nicht vermeiden. Ungünstig ist es, wenn wegen geringer Blutungen durch Anlegen des Esmarch'sehen Schlauches die ohnehin gefährdeten Theile der Circulation längere Zeit entzogen werden. - Die Schaffung reiner aseptischer Verhältnisse wïrde sich immer zu einem grosser und oft nicht absehbaren Eingriff gestalten; ausgedehnte Blosslegung und Entfernung aller gequetschten Partien wäre Grundbedingung; 
in vielen Fällen würde primäre Amputation oder Exarticulation nothwendig werden, die vielleicht bei conservativem Vorgehen vermieden werden könnte; gewiss ist ein so grosser primärer Eingriff bei dem shokirten Patienten nicht unbedenklich. - Liegt der Fall so, dass eine primäre Amputation unvermeidlich scheint, so kann eine versuchte Continuitätsresection bei gentigender Gefässerhaltung von Erfolg begleitet sein. - In 2 Fällen von Schuss-, beziehungsweise Masehinenverletzung des Unterarmes gelang Herrn Geheimrath Bardenheuer die Erhaltung der unversehrten Hand durch ausgedehnte Resection in der Mitte des Unterarmes; bei einem dritten Fall von Pferdebahnüberfahrung des Unterschenkels wurde die Amputation wegen Gangrän des Fusses nothwendig; es war in diesem Falle die Art. tibialis post. nachträglich thrombosirt. - Grössere Anwendung findet dieses Vorgehen bei complicirten Mittelhandverletzungen. - Die complicirten Wundrerletzungen der grossen Röhrenknochen sind so mannigfaltige, sie bieten im einzelnen so grosse Verschicdenheiten, dass eine schematische Vorschrift für die Behandlung: selbst an einem Krankenhaus nicht in Frage kommen kann. Indessen lassen sich doch allgemeine Gesichtspunkte fixiren. Gegentiber stehen sich zwei Anscbauungen, soll man primär spalten und die Wundfläche einer Revision unterwerfen, oder soll man möglichst conservativ verfahren. Dic Vertreter der ersteren Ansicht gehen davon aus, dass durch die Insulte bei der Verletzung und bis zum Eintritt in die klinische Behandlung wahrscheinlich Infectionskeime in die Wunde gelangt sind, die im retinirten Wundsecret, auf nekrotischen Fetzen einen vorzuggliehen Nährboden finden; sie wollen dieser Möglichkeit durch breite Wunddrainage begegnen. - Die andere Gruppe sieht gerade in der sofortigen Operation eine erneute Gefahr für die Schaffung. grösserer Wundflächen, durch die Unmöglichkeit, bei einfachem Spalten die Ausschaltung aller Taschen und Buchten und die Entfernung nekrotischer Theile zu bewirken; ferner durch die Möglichkeit einer Contactinfection. Dazu kommt die Wahrscheinlichkeit einer verzögerten Knochenconsolidirung und die Nothwendigkeit eines häufigen Verbandwechsels bei primärer Spaltung. Die thunlichste Vermeidung aller Manipulationen hat ihnen die besten Resultate ergeben.

Wir haben bisher ein durehschnittlich conservatives Verfahren geübt und im ganzen befriedigende Resultate erreicht. In Narkose wurden die Verletzungen einer äusseren Inspection unterzogen; unter Schutz der Wunde mit aseptischer Gaze wurde die Umgebung gereinigt; in die Wundhöhle wurde aseptische, in den letzten Jahren mit gelber Salbe bestrichene Gaze-Drainage eingeführt; dann folgte 
ein weit reichender Gypsverband; derselbe wurde nach 7 Tagen gefenstert und blieb im allgemeinen 4 Wochen liegen. - Geringe Abweichung boten die Fälle, in denen erst nach Spaltung der Haut eine Reposition eines vorstehenden Fragments möglich wurde; lagen grobe Verunreinigungen der aufgerissenen Weichtheile vor, so wurde mit Scheere und Pincette toilettirt. Vereinzclt wurde primäre Knochennaht unter streng aseptischem Vorgehen nothwendig, da sich die Reposition des dislocirten Humerussehaftes auf andere Weise nicht erlıalten liess; es betrifft 2 Fälle von Fract. complie. tumeri d. in der oberen Epiphyse, bezw. der Supra condylica, in denen der lange Schaft bis auf die Gelenkverbindung skelettirt war; der letzte Fall verlief mit verzögerter Heilung; der andere reactionslos. - Eine vergleichende Statistik der schweren complicirten Verletzungen der Extremitäten hat nur einen sehr bedingten Werth, da einerseits die relative Schwere der Verletzung, andererseits aber die individuelle Beurtheilung eine objective Deutung verhindert; sicher entschliesst sich der eine früher zur Operation, wo der andere den Versuch der Erhaltung wagen wird. Manchem wird ein Fall bekannt sein, bei welchem die nothwendig erachtete Amputation nicht zur Ausfluhrung $\mathrm{kam}$, sei es infolge Weigerung des Patienten, sei es, weil multipel schwere Verletzungen den Eingriff aufschieben liessen, - selbst Kähle und Bläue des peripheren Theiles haben uns nicht zur sofortigen Absetzung schwer verletzter Gliedmaassen bestimmt.

Ich kann über einen Fall berichten, der für denjenigen, der ihn frisch sah, in überraschender Weise den Werth einer conservativen Behandlung demonstirt. Der 30jährige Bremser Simonis gerieth nachts mit dem rechten Arm zwischen die Pufler zweier Waggons; es war ein Nothverband und Fsmarch'sche Binde angelegt worden; er wurde behufs Amputation dem Bürger-Hospitale überwiesen, 12 Stunden nach der Verletzung. - Am Unterarm bestand ein Hautdeffect der Beugeseite vom Carpus bis zum Ellbogen; die Haut war nach beiden Seiten bis auf eine schmale Zone circulär abgelöst; die gequetschte und theilweise zerrissene Beugemusculatur lag bis auf den Zwischenknochenraum bloss; dio diffuse Schwellung des Armes gestattete keine Orientierung ubber die Stellung der fracturirten Knochen; es bestand ausserdem Schwellung des ganzen Oberarmes und Bruch des Tuberc. maj. - Wir versuchten eine conservative Behandlung. Verband mit gelber Salbe nach Art der Mikulicz'schen Drainage; Lagerung auf eine Cramer'sche Schiene bei Festlegung des Schultergelenkes waren die einfachen Maassnahmen; der Verlauf war überraschend günstig; nachträglich wurde der grosse Hautdeffect geschlossen; eine Verbcsserung der verstellten Fracturenden versucht. - Die Knochenversehiebnng ist eine grosse geblieben; die Ulna ist fest consolidirt und mit dem oberen Randiusende durch Knochenbrücke verbunden; das untere Radiusfragment ist in mässigem 
Grade frei beweglich, gestattet dafür die theilweise Pro- und Supination der Hand. - Durch orthopaedische Nachbehandlung und besonders fleissiges Ueben hat der Mann einen funktionell sehr brauchbaren Arm behalten. - Patient ist sogar dienstfähig geworden; er coupirt zur Zeit die Billette am Bahnhof.

In anderen Fällen ermöglichte die conservative Behandlung bei ausgedehnten Hautverletzungen das Erhaltenbleiben grösserer oder kleinerer Hautinseln, welche eine secundäre Deckung des Defects sehr erleichterten; die nekrotischen Hautheile stiessen sich unter dem Salbenverband ohne Entzündung ab. - Dass anch Misserfolge nicht ausgeblieben sind, ist selbstverständlich; wir glauben dieselben aber weniger der Behandlung als der Verletzung zuschreiben zu mlissen. In 3 Fällen von complicirten Oberschenkelbrlichen bei Verletzung des Kniegelenkes wurde die secundäre Amputation nothwendig; dazu kommen zwei complieirte - comminutive Fracturen des Unterschenkels, die nach langwieriger Eiterung eine Amputation nicht umgehen liessen; von diesen starb einer an Allgemeininfection; ein zweiter starb an den Folgen multipler complic. Verletzungen. Gegentiber den stets gefährlichen complic. Verletzungen der grossen Röhrenknochen ist der Verlauf der complicirten peripheren Läsionen ein sehr viel günstigerer. Es handelt sich überwiegend um Verletzungen der Finger und Hände im maschinellen Betriebe; hauptsächlich verursacht durch Holzschneide- und Hobelmaschinen, durch Kammräder, Buchdruckerpressen und Walzen; dazu kommen Zehenabquetsehungen - durch Ueberfahren ete. - Die von solchen Maschinen gefassten Finger weisen multiple Verletzungen auf; ausser der Hautzerfetzung sind Sehnenscheiden und Gelenke eröffnet. Sehnen angeschnitten oder ganz durehtrennt; die Knochen sind in mannigfaltigster Weise, häufig gesplittert fracturirt. Einen Unterschied bilden die mehr dureh schneidende Instrumente verursachten Verletzungen gegenüber den complicirten reinen Quetschungen, als deren Prototyp Kammradverletzungen anzusehen sind. - Bei ersteren sind die Wundflächen wenigstens theilweise glatt. Die Finger zuweilen bis auf kleine Hautbrticken durchtrennt; bei letzteren die Finger und Hände förmlich zerhackt, dazu von der Arbeit und den geölten Maschinentheilen besudelt. - Präsentiren sich diese Art von Verletzungen auch als bedenkliche, so fehlen ihnen doch die gewaltige Shocwirkung der grossen complicirten Verletzungen und deren colossale Resorptionsflächen; wir finden bei den peripheren Maschinenverletzungen eine Abquetschung der resorbirenden Lymph- und Blutgefässe und Fehlen erheblicher Blutung; noch crhaltene Weichtheil- 
brïeken bieten bei den gïustigen Cireulationsverhältnissen Chancen für die Erhaltung gefährdeter peripherer Theile.

Solche Verletzte kamen gewöhnlich mit einem provisorischen Verband aus den Verbandskästchen der Fabriken bedeckt sofort von der Unglüeksstelle in's Hospital. - Bis zum vorigen Jahre wurden dieselben nach Application cines halbstüdigen 2proc. Soda- oder $1 / 2$ pro mille Sublimatbades in Narkose gründlich gereinigt und dann einer primären Wundtoilette nnterworfen. - Dabei konnte es nicht ausbleiben, dass ganze Finger- oder Theile derselben geopfert wurden, die wir bei abwartender Methode vielfach sich erhalten sehen. - Wenn wir uns auch bei peripheren Fussverletzungen leicht entschliessen könnten, schwer lädirte Zehen oder den Vorderfuss primär abzusetzen in der Gewissheit, ein relativ gutes funktionelles Resultat in kürzester Zeit zu erzielen, gilt dies gewiss für die Verletzungen der Finger nicht in gleicher Weise. - Die Erwägung, dass in einzelnen Fällen nachträylielı die Entfernung eines die Gebranchsfähigkeit störenden Fingers oder Theile desselben nöthig werden, kann uns nicht bestimmen, radical vorzugehen, zumal wir auch bei primärer Wundtoilette gequetschter Theile auf eine primäre Heilung verzichten. - Wie der Werth der einzelnen Finger verschieden, so kann unter Umstïnden die Erhaltung eines für die Erwerbsfähigkeit unbrauchbaren oder störenden Fingers seln whinschenswerth sein. Betrifft aber die Verletzung den Daumen oder die sämmtlichen übrigen Finger, so kann durch Erhaltenbleiben einzelner Theile wescntlicher Vortheil für die Funktion geschaffen werlen.

Seit Frühjahr 1897 wurden bei uns 22 Fälle complicirter Fingerund Handverletzungen stationär einer rein conservativen Behandlung. unterzogen; es sind dies diejenigen Fälle, in denen mindestens 2 Finger ausgedehnte Quetschungen durch Maschinengewalt erlitten hatten, betroffen waren sechszehn jüngere Personen beiderlei Geschlechts im Alter von 14-26 Jahren, sechs betrafen erwachsene Männer; dazu kommen 3 Fälle von Zehenabquetschungen. Ueber die Ausdehnung der einzelnen Verletzungen zu berielıten, würde zu weit führen. Wir haben thunlichst bei frisch cingelieferten Fällen photographische Aufnahmen gemacht, immer aber wurde nach Ablauf der Behandlung. eine Röntgen-Photographie angefertigt. Herr Assistenzarzt Dr. Wild t hat sich in dankenswerther Weise um die Anfertigung dieser Bilder verdicnt gemacht. - Ich erlaube mir, Ihnen einige cliarakteristische Photographien herumzugeben; davon interessirt vielleielit die Bildergrippe der Patientin M., an der ich Ihnen den Verlauf eines ganz abwartenden Verfahrens demonstriren kann; - ich bemerke, 
dass das Abwarten eines secundären Eingriffes aus nahe liegenden Grinden zu Demonstrationszwecken verschoben wurde. Patientin hatte dureh eine Buchdruckermaschine eine ausgedehnte Weichtheilquetschung der linken Hand mit Verletzung der Beugesehnen des 3.-5. Fingers und complicirte Fracturen des 2.-5. Fingers erlitten; der Verlauf war, wie in allen 22 Fällen von Masclinenverletzung, durchaus günstig; am 1. Tag leichte Temperatursteigerung, in den ersten Tagen Schmerzen, von da ab reactionslos. - Es besteht heute neben der Schwellung der Hand eine kleine Fistel an der Basalphalange des 3. Fingers, der am schwersten getroffen war und eine trockene Gangrän der Fingerkuppe des 4. Fingers. Bewegungstibungen der Finger sind erst begonnen worden. - Viel charakteristischer präsentiren die Nützlichkeit eines abweichenden Verfahrens folgende Bilder. Sie sehen in dem Falle Höhn (Hobelmaschinenverletzung) an der ersten Aufnahme, die im Verband in supinirter Stellung gemacht wurde, die Schwere der Verletzung; gesplitterte Längsfracturen des Index, complicirte Splitterfracturen der I/II Phalange des 3. und 4. Fingers, die Zermalmung der Basalphalange des kleinen Fingers und Verletzung des Interphalangealgelenkes II/III an demselben Finger. Diese Photographie, 4 Monate später, zeigt die Erhaltung sämmtlicher Finger; eine secundäre Operation wurde nicht vorgenommen.

Besondere Aufmerksamkeit verdienen die beiden Fälle Berge r und Lepping; im ersteren Falle sehen wir neben der Amp. violent. dig. II und V am 3. Finger die centrale Hälfte der 2. Phalange erhalten und einem peripheren Theil der letzten Phalange, in gleicher Weise am 3. Finger mit dem Unterschied, dass 3 Knochenstäckchen der letzten Phalange vorhanden sind; ähnliches zeigt der 4. Finger im Fall Lepping. - Auch in diesen beiden Fällen war eine Nachoperation nicht nothwendig. Der Wundverlanf gestaltete sich durchaus gtinstig; in einem Falle kam eine Temperatursteigerung von $38,5^{0}$ vor; eine geschwollene Cervicaldrüse zeigte an, dass eine Resorption infectiösen Stoffes stattgefunden hatte. - Während zuerst der Vorsicht halber die Wunden nach 24 Stunden einer Revision unterzogen wurden, lassen wir seitdem bei diesen Verletaungen den 1. Verband durchschnittlich 10 Tage liegen, vorausgesetzt, dass die Temperatur normal geworden ist, keine Schmerzhaftigkeit oder sonstige Störung vorliegt. - Der 1. Verbandwechsel wurde schonungshalber im Sublimatbade vorgenommen; es präsentirte sich oft schon beim 1. Verbandwechsel eine gut gereinigte Granulationsfläche; angerissene Sehnen, Knochensplitter wurden nicht entfernt; der Circulation ganz entzogene Theile 
zeigen demarkirte Gangrän ohne Reaction; zumeist mumificirt; indess wurde zweimal echte Fäulniss in abgestorbenen Fingern constatirt, ein Beweis, dass wir bei der Wertlıschätzung des conservativen Verfahrens das Antisepticum nicht ïberschätzen dïrfen; es folgte ein 2. Verband; darnach konnten corrigirende Eingriffe vorgenommen werden; dieselben waren indess nicht so liäufig, wie man vermuthen sollte; auch in den Fällen, wo die mögliche Erhaltung lädirter Finger für die Erwerbsfähigkeit von zweifelhaftem Werth erschien, zeigte sich uns ein zunächst abwartendes Verfahren als das schonendste; es ist natürlich auch hierbei auf den einzelnen Fall Rücksicht zu nehmen, und hat es selbstverständlich keinen Zweck, die zermalmte Fingerkuppe eines 4. Fingers, z. B. bei einem Arbeiter, einer mehrwöchentlichen conservativen Behandlung: zu unterziehen; er muss eben individualisirt werden; - während für eine Dame die Erhaltung eines verletzten Fingers von hohem Werth sein kann, ist bei dem Arbeiter die möglichst vollständige und frülızeitige Erwerbsfähigkeit maassgebend.

So gut unsere Erfahrungen unter den gewöhnlichen Verhältnissen bei complicirten Wundverletzungen mit dem conservativen Verfahren gewesen sind, so liaben wir uns doch die Frage vorzulegen, ob wir durch ein solches Vorgehen die Infection mit Tetanus begtinstigen. - Wir haben in den beiden letzten Jahren 2 Fälle von Wundtetanus zu verzeichnen; es ist statistisch nicht mehr wie im Durehschnitt des letzten Jalırzehntes.

Der 1. Fall betraf einen 15jährigen Knaben, der mit dem Arm in eine Hechelmaschine für ungereinigten Flachs geraten war; Patient wurde eine Stunde post Verletzung mit einer klaffenden Risswunde des rechten Oberarmes eingebracht; die Wunde reichte von der Achsel bis oberhalb des Ellbogens, die Haut war weit abgelöst, das Gefäss und und Nervenbündel lag sichtbar in der Wunde, Pulsation der Brachialis war sichtbar, der ladialpuls war nicht zu fühlen; die Musculatur des Biceps und Barchial. war gequetseht olne starke Zerreissung, eine grobe Wundvereinigung lag nicht vor. Die weit abgelöste Haut ermöglichte eine ausgiebige Wunddrainage; der Verlauf schien auch anfangs günstig; am 2. Tag Temp.-Steigerung $38,5^{\circ}$; Patient klagte wenig; die anfangs kühle Hand war wärmer geworden und nicht geschwollen; Bewegungen wurden in geringem Grade ausgeführt, die Sensibilität war nahezu erloschen. Am 6. Tage zeigte die Wunde, welche stark secernirt hatte, ein gutes Aussehen der Musculatur: die N. medianis und ulnaris waren in grosser Austehnung gangränös, die Brachialis war thrombosirt; das Aussehen der linterarme und der Haud war ein normales. Am 10. Tag klagte der Patient über Zuckungen im verletzten Arm, die anderen Tages angeblich geschwunden waren; es begannen leichte abendliche Temperatursteigerungen; am 12. Tage beginnender Trismus und Schluckbeschwerden; 
rasche Steigerung der Symptome; am 16. Tag Exitus. Die Wunde batte ein so gutes Aussehen, dass die Exarticulation erst nach der vergeb-

Fig. 1.

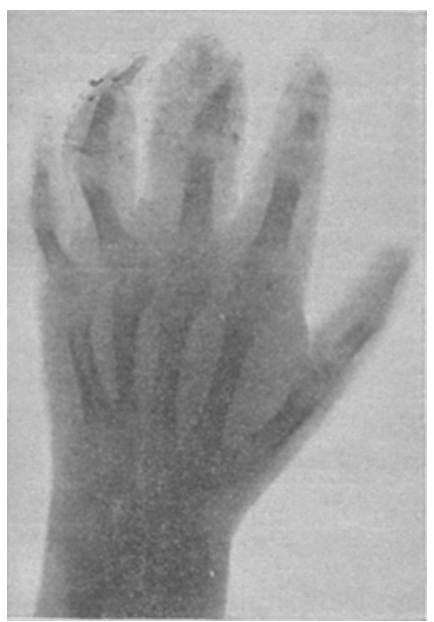

Complicirte multiple Knochenverletzungen des 2. bis 5. Fingers der rechten Hand (Buchdruckerpresse) bei 14 jilihr. Mïdchen PetronellaMay. Periphere truckene Nekrose am 3. und 4. Finger.

Fig. 2.

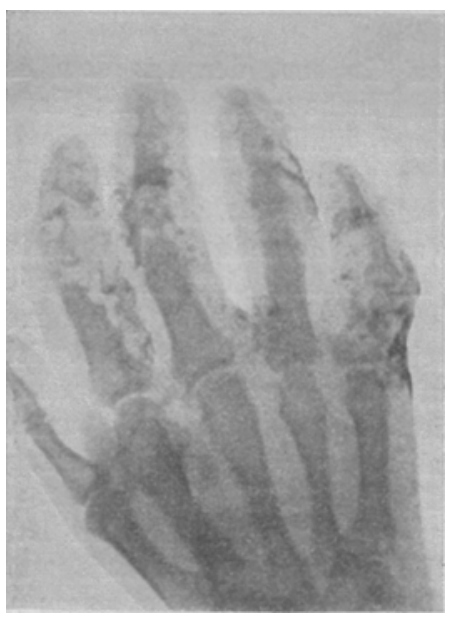

lichen Anwendung von T. Antitoxin ausgeführt wurde, allerdings auch ohne Erfolg. $\mathrm{Ob}$ in diesem Falle eine primäre Wundtoilette den unglïcklichen Ausgang vermeiden lässt, ist fraglich, da die Ausdehnung der Quetschung nicht abzusehen war, und bei den vorliegenden Verhältnissen eine primäre Exarticulation nicht berechtigt schien. Bedenklicher ist der 2. Fall, der allerdings erst nach 24 Stunden in unsere Behandlung trat; es handelte sich um eine Abquetschung der 1. Zehe und Quetschung der 2. und 3. ausserdem kleine Hautwunden; verursacht durch Ueberfahrung von einer Karre, mit der vorher Mist gefahren war. Die Wunde wurde mit einem typischen Salbenverband versehen und in einer Volkmann'schen Schiene gelagert. Die ersten Tage verliefen ohne Störung; am 4. Tage geringe Temperaturerlöhung; am 6. Tage
Fig. 3.

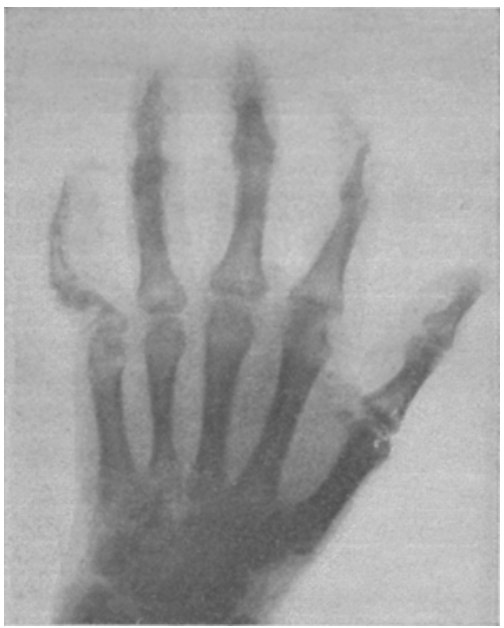

Complicirte multiple Knochenverletzungen mit Splitterungen vom 2. bis 5. Finger der linken Frand durch Hobelmasehine bei 32 jähr. Joh. Hähn.

Fig. 2. Nach dem 1. Verband.

Fig. 3. Nach der Heilung. Kein sccundürer Eingriff. 
beginnende Schluckbeschwerden; die Symptome nahmen rasch $z$ u; trotz Absetzung des verletzten Vorderfusses, der in der Umgebung keine Spur einer phlegmonösen Entzuinduug bot, trotz Antitoxininjectionen verlief der Fall foudroyant; in der Nacht vom 7.-S. Tage Exitus. - Der bakteriologische Befund wird noch von dem hiesigen bakteriologischen Institut aus veröffentlicht werden. - Der ausserordentlich stiirmische Verlauf dieses Falles deutet darauf hin, dass eine schwere Infection stattgefunden hatte, die auch dureh einen Eingriff nach 24 Stunden vielleicht nicht mehr aufzuhalten war; andererselts ist es aber auch wahrscheinlich, dass die Tetanussporen in dem nekrotischen, der Circulation entzogenen Gewebe den günstigen Nährboden zur Entwickelung einer hohen Virulenz gefunden haben.

Fig. 4.

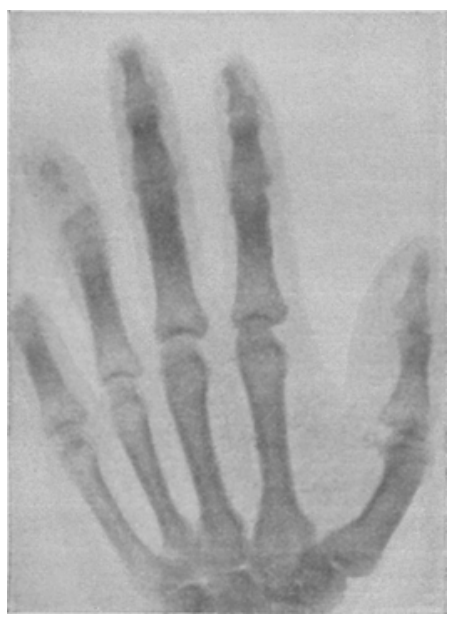

Complicirto Zerreissung des 3 . bis 5 . Wingers der linken ITand durch llobelmaschine bei 45 jithr. With. Malzer. Atm 4. Finger ist von der II. Phalange die contrale Hälfte, von der III. I'halange sind einzelne periphere Knochentheilchen erhalten. Kein seeundärer Kingrifif.
Fig. 5.

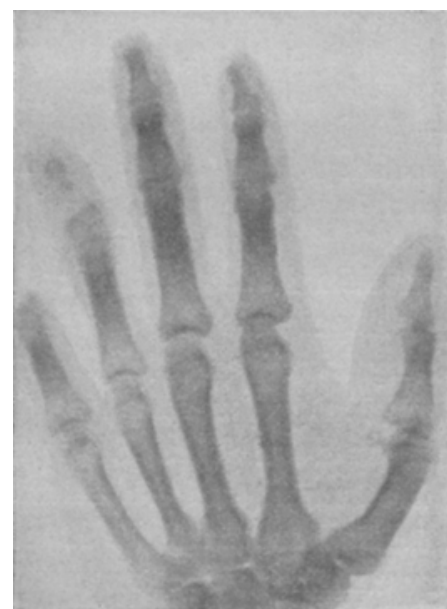

Multiple complicirte Verletzunen des 2. his 5. Fingers der linken Iland durch Holzschneidemaschine bei 24 juhr. Herm. Lipping.

Am 4. und 3. Finger ïhnliche Verbält niase wie im Fall 4 . Kein secundärer Eingriff.

Dieses Vorkommniss wird uns veranlassen, der Entstehungsursache der Verletzung besondere Aufmerksamkeit zu widmen und bei Beschmutzungen einer Wunde mit Erde, Mist ete. grtundliche Wundtoilette vor\%unebmen. - Gegeuiiber diesen Ausnahmefällen hat uns die Beobachtung" eines grossen Materials an Verletzungen aller trt die Berechtigung und Vorzüge einer weitgehenden conservativen Behandlung unter gewöhnlichen Verhältnissen bewiesen. - Grundsatz muss bei den complicirten Wundrerletzungen sein, unter möglichst geringen Opfern diesen sohwierigen Verhältnissen zu begegnen. 